\title{
Surgical Outcomes of Microscopic versus Unilateral Biportal Endoscopic Decompression for Lumbar Spinal Stenosis: A Preliminary Report
}

\author{
Jun Hong Lee, Sung Ik Cho, Chung Kee Chough \\ Department of Neurosurgery, Yeouido St. Mary's Hospital, The Catholic University of Korea, Seoul, Korea
}

\begin{abstract}
Objective: The objective of this study was to compare clinical outcomes and length of hospitalization between microscopic decompression and unilateral biportal endoscopic (UBE) decompression for lumbar spinal stenosis.

Methods: Medical records of patients with lumbar spinal stenosis who underwent microscopic fenestration and unilateral laminotomy bilateral decompression (ULBD) via UBE from January 2017 to May 2019 were reviewed retrospectively. Clinical outcomes were reviewed regarding preoperative and postoperative visual analogue scale of back pain (VAS-B), visual analogue scale of leg pain (VAS-L) and Oswestry Disability Index of the lumbar spine (ODI) scores. Length of hospitalization, postoperative total drainage volume, and type and incidence of complications were also reviewed.

Results: A total of 103 patients (44 microscopic fenestration and 59 ULBD via UBE) was selected. The mean age of the two groups was the same. The preoperative and postoperative clinical results of the two groups showed no significant difference, and the mean operation time and complications were similar in the groups. The microscopic fenestration group (10.37 days) had longer hospitalization than the ULBD via UBE group ( 7.53 days, $p=0.01$ ) and larger postoperative drainage volume $(157.10 \mathrm{cc})$ than the ULBD via UBE group $(83.50 \mathrm{cc}, \mathrm{p}<0.01)$.

Conclusion: These two surgical procedures showed no significant difference in clinical outcome, but the ULBD via UBE group had shorter hospitalization, allowing patients to return to their daily lives earlier. These improved recovery after surgery suggests that ULBD via UBE could be a viable surgical option for geriatric patients with lumbar spinal stenosis.
\end{abstract}

Key Words: Biportal endoscopic, Decompression, Lumbar stenosis, Microscopic, Minimally invasive surgery

\section{INTRODUCTION}

Lumbar spinal stenosis is a clinically symptomatic condition caused by narrowing of the spinal canal that compresses nerve roots $^{1,14)}$. Most elderly people experience at least one episode of lower back pain, sciatica, claudication, or walking difficulty during their lives ${ }^{3)}$. Previous studies have reported that surgery is required when symptoms are refractory to medical treatment or combined with neurological deficits ${ }^{9,11,14,16}$.

Surgical management of lumbar spinal stenosis has changed in the last decades. In the 1970s, use of the microscope and specially designed retractors gave rise to modern spinal sur-

Corresponding author: Chung Kee Chough, MD, PhD Department of Neurosurgery, Yeouido St. Mary's Hospital, The Catholic University of Korea, 10, 63-ro, Yeongdeungpo-gu, Seoul 07345, Korea

Tel: +82-2-3779-1 187, Fax: +82-2-786-5809

E-mail: chough@catholic.ac.kr gery $^{4,5)}$. Currently, minimally invasive decompressive surgery, such as microscopic decompression and unilateral laminotomy bilateral decompression (ULBD), is considered routine with standard minimally invasive surgery for lumbar spinal steno$\operatorname{sis}^{9,14,16}$.

In the 1980s, lumbar discectomy using endoscopy was introduced. Publication of Kambin's anatomical safe triangle fostered great progress in endoscopic spine surgery ${ }^{7,15)}$. Minimally invasive surgery using endoscopy for lumbar spinal stenosis was introduced quickly with the relatively recent development of the unilateral biportal endoscopic (UBE) appro$\mathrm{ach}^{13)}$. This endoscopic approach was based on the same operative technique as other surgical procedures, such as ULBD, ${ }^{9,13)}$. The UBE was performed through two separate, small surgical incisions, so surgeons could achieve precise decompression in a clear and magnified surgical field with limited damage to adjacent structures ${ }^{1,11,12,14)}$. To date, there are many reports of UBE performance and radiological results, as seen in Park et al. and Heo et $\mathrm{al}^{8,13)}$. But there are only a few papers that 
compare the clinical results of conventional microscopic decompression and UBE. Thus, we conducted this study to compare outcomes of these two surgical methods and feasibility of ULBD via UBE for surgical treatment of geriatric patients.

\section{MATERIALS AND METHODS}

\section{Patient Selection and Study Design}

We retrospectively reviewed patients who underwent onelevel lumbar microscopic fenestration or ULBD via UBE from January 2017 to May 2020. There were 16 men and 28 women in the microscopic fenestration group and 30 men and 29 women in the ULBD via UBE group (Table 1). The mean age at time of the surgery was $69.11 \pm 8.58$ years in the microscopic fenestration group and $69.64 \pm 11.05$ years in the ULBD via UBE group. All patients presented with leg pain with or without lower back pain. Inclusion criteria were as follows: (1) One-level surgery for central canal stenosis, (2) microscopic fenestration or UBE surgery, and (3) a minimum of three months follow-up. Exclusion criteria were as follows: (1) multilevel surgery, (2) follow-up loss before three months, (3) other spinal pathologic conditions with or without stenosis.

\section{Operative Technique}

After induction of general anesthesia, the patients were placed prone, with the abdomen free, over the Wilson-frame. The surgical field was prepared in a sterile manner.

For the microscopic fenestration group, the surgical procedure is almost identical to the description of Hatta et al. and is as follows ${ }^{6}$. For one-level decompression, a 30-mm midline skin incision was made, centered at the interspinous level. The muscles and periosteum were detached together from the bone. The caudal half of the upper spinous process/lamina and the cranial third of the lower spinous process/lamina were exposed. A surgical microscope was used after exposure of the interlaminar space. The surgical field was gradually expanded by retracting the split ligaments and bilateral paravertebral muscles laterally using a Gelpi self-retaining retractor. After removal of the caudal part of the upper spinous processes, the cranial third of the lower lamina was removed to free the caudal margin of the ligamentum flavum by drill. After partial laminotomy of the caudal half of the upper lamina, a dome-like expansion was performed by removing the inner laminar plate to the location where the cranial margin of the ligamentum flavum is freed, the bilateral facet joint was undercut to expose the lateral margin of the ligamentum flavum, then the ligament was removed using a Kerrison punch. Central canal and nerve root canal decompression was accomplished by trimming the medial margins of the upper and lower articular processes together with the capsular portion of the ligamentum flavum flush with the medial border of the pedicle bilaterally. After decompression was complete, meticulous hemostasis and layer-by-layer closure with drain were performed.

The other group underwent ULBD via UBE under continuous normal saline irrigation ${ }^{9}$. In the same manner of microscopic decompression, the spinal levels were determined and marked on the skin. UBE decompression requires two small incisions through the deep fascia; one for endoscope viewing and continuous irrigation, the other one for outflow of saline and instrument portal ${ }^{11}$. An arthroscopic system with either a 0 - or 30-degree scope is essential. The target area for decompression was at the spino-laminar junction, the intersection between the lower lamina margin and a line $1 \mathrm{~cm}$ lateral to the spinous process (we preferred a left-sided approach for a right-handed surgeon $)^{9}$. A K-wire was introduced in the di-

Table 1. Demographics of the two subgroups

\begin{tabular}{|c|c|c|c|}
\hline & & Microscopic fenestration & Unilateral laminotomy bilateral decompression via unilateral biportal endoscopy \\
\hline \multirow[t]{2}{*}{ Sex } & Male & 16 & 30 \\
\hline & Female & 28 & 29 \\
\hline Age (range: $38-90)$ & & $69.11 \pm 8.58(33-90)$ & $69.64 \pm 11.05(51-87)$ \\
\hline \multirow[t]{4}{*}{ Level } & $\mathrm{L} 2 / 3$ & 2 & 0 \\
\hline & $\mathrm{L} 3 / 4$ & 12 & 16 \\
\hline & $\mathrm{L} 4 / 5$ & 29 & 40 \\
\hline & L5/S1 & 1 & 3 \\
\hline Previous Hx & & 9 & 8 \\
\hline
\end{tabular}

Values are mean \pm SD. 
rection of the target. Following removal of the dilators, an RF wand was inserted. Interlaminar soft tissue was dissected laterally to the medial margin of the facet capsule. After laminotomy at the spino-laminar junction, the ipsilateral ligamentum flavum was removed until full mobilization of the lateral border of the nerve root was achieved ${ }^{2,13}$. Complete removal of the contralateral ligamentum flavum and sublaminar decompression were performed using a Kerrison punch and curette. Contralateral decompression was performed until the contralateral traversing nerve root was identified and decompressed. The degree of decompression was assessed by normal respiratory-induced dural pulsation and confirmed with endoscopic viewing ${ }^{9,16}$. Meticulous hemostasis and closure of twoportal incision with drain were achieved.

\section{Statistical Analysis}

Statistical analysis was performed using SPSS statistical software package 26.0 for Windows (SPSS Inc, Chicago, IL, USA). The independent $t$-test and chi-square test were used to analyze differences and evaluate statistical significance, which was set at $\mathrm{p}=0.05$.

\section{RESULTS}

The clinical parameters are summarized in Table 2. Mean preoperative visual analogue scale of back pain (VAS-B) and visual analogue scale of leg pain (VAS-L) and the ODI of the lumbar spine scores in the microscopic fenestration group were $5.64 \pm 3.20,7.11 \pm 3.20$, and $49.84 \pm 20.86$, respectively (Table 2). These values in the ULBD via UBE group were $5.02 \pm 2.94,6.42 \pm 2.37$, and $44.40 \pm 18.37$, respectively. One month after surgery, mean VAS-B, VAS-L, and ODI scores in the microscopic fenestration group were $1.50 \pm 1.09,1.25$ \pm 1.06 , and $16.50 \pm 7.42$, respectively. In the ULBD via UBE group, these values at one month after surgery were 1.56 \pm 1.37, $1.44 \pm 1.33$, and $17.66 \pm 8.62$, respectively. Three months after surgery, mean VAS-B, VAS-L, and ODI scores in the microscopic fenestration group were $1.18 \pm 0.76,1.05 \pm 0.91$, and $14.64 \pm 4.33$, respectively, while those in the ULBD via UBE group were $1.32 \pm 1.51,1.32 \pm 1.38$, and 16.41 \pm 8.67 .

For clinical outcome, the microscopic fenestration group had no significant difference from the ULBD via UBE group in preoperative VAS-B (5.64 versus $5.02, \mathrm{p}=0.31$ ), preoperative VAS-L ( 7.11 versus $6.42, \mathrm{p}=0.18$ ), and preoperative ODI (49.84 versus $44.40, \mathrm{p}=0.16)$ scores. At one month after surgery, there was no significant difference between the groups in VAS-B (1.50 versus $1.55, \mathrm{p}=0.81)$, VAS-L $(1.25$ versus $1.44, \mathrm{p}=0.44)$, and ODI $(16.50$ versus $17.66, \mathrm{p}=0.48)$ scores. Similarly, at three months after surgery, there was no significant difference between the microscopic fenestration and ULBD via UBE groups in VAS-B (1.18 versus $1.32, \mathrm{p}=$ $0.54)$, VAS-L (1.05 versus $1.32, p=0.23)$, and ODI (14.64 versus $16.40, \mathrm{p}=0.22)$ scores.

Table 2. Perioperative associated clinical parameters between surgery groups

\begin{tabular}{lccc}
\hline \hline & Microscopic fenestration & $\begin{array}{c}\text { Unilateral laminotomy bilateral decompression } \\
\text { via unilateral biportal endoscopy }\end{array}$ & p-value \\
\hline Operation time (min) & 107 & 104 & 0.61 \\
Total drain output (cc) & 157.10 & 83.50 & $<0.01^{*}$ \\
Length of hospitalization (days) & 10.37 & 7.53 & 0.01 \\
Preop VAS-B score & 5.64 & 5.02 & 0.31 \\
Preop VAS-L score & 7.11 & 6.42 & 0.18 \\
Preop ODI score & 49.84 & 44.40 & 0.16 \\
1 m VAS-B score & 1.50 & 1.55 & 0.81 \\
m VAS-L score & 1.25 & 1.44 & 0.44 \\
m ODI score & 16.50 & 17.66 & 0.48 \\
3 m VAS-B score & 1.18 & 1.32 & 0.54 \\
3 m VAS-L score & 1.05 & 1.32 & 0.23 \\
m ODI score & 14.64 & 16.40 & 0.22 \\
\hline
\end{tabular}

"Statistically significant differences (p-value $<0.05$ )

VAS-B: Visual analogue scale of back pain, VAS-L: Visual analogue scale of leg pain, ODI: Oswestry disability index, Preop-VAS-B: Preoperative VAS-B, 1m VAS-B: VAS-B at 1 month after surgery, $3 \mathrm{~m}$ VAS-B: VAS-B at 3 months after surgery. 
Table 3. Demographics of complications between surgery groups

\begin{tabular}{lcc}
\hline \hline Complication & Microscopic fenestration & Unilateral laminotomy bilateral decompression via unilateral biportal endoscopy \\
\hline Dura tear (cases) & 4 & 6 \\
Infection & 2 & 2 \\
Postoperative epidural hematoma & 1 & 1 \\
Incomplete decompression & 0 & 2 \\
Others & $1^{*}$ & $1^{* * *}$ \\
Total (p-value=0.78) & 8 & $1^{* * *}$ \\
\hline
\end{tabular}

"The facet cystic change in the epidural space.

"The temporary right L5 nerve root palsy.

Other data showed no significant difference between microscopic fenestration and ULBD via UBE. Operation time of microscopic fenestration and ULBD via UBE groups were 107 and 104 minutes, respectively $(\mathrm{p}=0.61)$. Also, the respective numbers of complications in the microscopic fenestration and ULBD via UBE groups were 8 and $12(\mathrm{p}=0.78)$. Complications in the microscopic fenestration group consisted of dura tear, infection, postoperative epidural hematoma, and facet cystic change in the epidural space (Table 3). Dura tear was the most common complication (4 cases). In the ULBD via UBE group, complications were dura tear, infection, postoperative epidural hematoma, incomplete decompression, and temporary right L5 nerve root palsy. The most common complication was the dura tear (6 cases).

The groups showed significantly different length of hospitalization, with longer hospitalization in the microscopic fenestration group (10.37 days) than in the ULBD via UBE group ( 7.53 days, $\mathrm{p}=0.01$ ). Post-operative drainage volume of the microscopic fenestration group was larger $(157.10 \mathrm{cc})$ than that of the ULBD via UBE group $(83.50 \mathrm{cc}, \mathrm{p}<0.01)$.

\section{DISCUSSION}

Traditionally, open microsurgical decompression including microscopic decompression has been the gold standard treatment for lumbar spinal stenosis ${ }^{3,9,15,16}$. In recent years, endoscopic spinal surgery for lumbar spinal stenosis has been introduced and progressed ${ }^{1,10,11,13,14)}$.

In our results, there was no statistically significant difference in VAS-L, VAS-B, or ODI scores at one month or three months follow-up. In meta-analysis of Pranata et al., there was no difference in VAS and ODI at postoperative follow-up between biportal endoscopic and microscopic techniques for lumbar spinal stenosis ${ }^{1,10,14)}$. Most previous studies used microscopic ULBD without microscopic fenestration. More studies are needed for accurate comparison of clinical outcomes of a microscopic decompression group and a UBE group.

The most common complication was dura tear. Heo et al. and Park et al. reported the same results ${ }^{8,13)}$. Complications can be reduced with operator's experience and depend on the proficiency of the operator.

In most studies, there was significant difference in length of hospitalization ${ }^{1,10,11,14)}$. In our study, there was longer hospitalization in the ULBD via UBE group (11.091 days) than in the microscopic fenestration group (17.276 days, $\mathrm{p}=0.54$ ). But there were three statistical outliers who remained in the hospital for 514, 66, and 42 days (2.5th percentile=less than 5 days, 97.5 percentile $=$ more than 28 days). Two of these patients were in the ULBD via UBE group, and the other was in the microscopic fenestration group. After excluding the outliers, there was statistically significant difference in length of hospitalization, with that of the ULBD via UBE group shorter (7.53 days) than that of the microscopic fenestration group (10.37 days, $\mathrm{p}=0.01$ ). Similarly, the endoscopic procedure significantly reduced postoperative drainage volume in our study. Hemo-vac drainage in milliliters previously was reported to be lower in the UBE group ${ }^{10,14)}$.

Based on these results, the ULBD via UBE group was superior in time from surgery to returning to daily life ${ }^{10,14)}$. Regardless of surgical method, there was no significant difference in clinical outcome, operation time, and incidence of complications. Considering that the endoscopic surgery was an early result in an ongoing learning curve, ULBD via UBE was not inferior to conventional microscopic decompression ${ }^{1,9,11,13,14)}$. Every complication was reviewed in the early period of resea$\mathrm{rch}$, and the number gradually decreased as proficiency increased $^{13,14)}$

In our study, the mean age at time of the surgery in the ULBD via UBE group was 69.64 years and the elderly patients aged 70 or older accounted for 54.23 percent of the group. 
In these elderly patients, the ULBD via UBE showed comparable results to microscopic fenestration. The advantages that the postoperative drainage volume is low and rapid discharge is possible are able to allow the patients to avoid complications followed by extended immobilization. Furthermore, It can be performed with local anesthesia, if general anesthesia is difficult due to the comorbidity of the patient. Therefore, the ULBD via UBE is considered suitable surgical treatment of lumbar spinal stenosis in geriatric patients.

There were some limitations in this study. The number of cases was small, and the follow-up period was relatively short (3 months). Also, VAS and ODI score are subjective to estimate clinical outcome and do not consider motor weakness. We recommend researchers examine this topic with more objective and various criteria for estimating outcomes. Finally, this study was retrospective, so the level of evidence was relatively low. Thus, large, prospective, and long-term studies are needed to compare the outcomes of microscopic fenestration and ULBD via UBE. We anticipate additional experience and data to enable accurate judgments about pros and cons of UBE.

\section{CONCLUSION}

The ULBD via UBE group showed shorter hospitalization and less postoperative total drainage volume compared with the microscopic fenestration group. There was no significant difference in operation time, complications, and clinical outcome. In other words, these two surgical procedures have no significant difference in clinical outcome, although the UBE group is able to return to their daily lives earlier. This study suggests endoscopic spinal surgery as a good alternative to open surgery.

\section{REFERENCES}

1. Chen T, Zhou G, Chen Z, Yao X, Liu D: Biportal endoscopic decompression vs. microscopic decompression for lumbar canal stenosis: A systematic review and meta-analysis. Exp Ther Med 20:2743-2751, 2020

2. Choi CM, Chung JT, Lee SJ, Choi DJ: How I do it? Biportal endoscopic spinal surgery (BESS) for treatment of lumbar spinal stenosis. Acta Neurochir (Wien) 158:459-463, 2016

3. Djurasovic M, Glassman SD, Carreon LY, Dimar JR, $2^{\text {nd }}$ : Contemporary management of symptomatic lumbar spinal stenosis. Orthop Clin North Am 41:183-191, 2010
4. Grochulla F: Surgical microscopy in spinal surgery in vieweg $\mathrm{U}$, Grochulla $\mathrm{F}$ (eds): Manual of spine surgery. Berlin, Heidelberg: Springer Berlin Heidelberg, 2012, pp43-47

5. Hamawandi SA, Sulaiman, II, Al-Humairi AK: Open fenestration discectomy versus microscopic fenestration discectomy for lumbar disc herniation: A randomized controlled trial. BMC Musculoskelet Disord 21:384, 2020

6. Hatta Y, Shiraishi T, Sakamoto A, Yato Y, Harada T, Mikami $\mathrm{Y}$, et al.: Muscle-preserving interlaminar decompression for the lumbar spine: A minimally invasive new procedure for lumbar spinal canal stenosis. Spine (Phila Pa 1976) 34:E276-280, 2009

7. Hausmann B, Forst R: Shaving of the lumbar disk space - A new technique in lumbar nucleotomy. Archives of Orthopaedic and Traumatic Surgery 103:284-285, 1984

8. Heo DH, Lee DC, Park CK: Comparative analysis of three types of minimally invasive decompressive surgery for lumbar central stenosis: Biportal endoscopy, uniportal endoscopy, and microsurgery. Neurosurg Focus 46:E9, 2019

9. Hwa Eum J, Hwa Heo D, Son SK, Park CK: Percutaneous biportal endoscopic decompression for lumbar spinal stenosis: a technical note and preliminary clinical results. J Neurosurg Spine 24:602-607, 2016

10. Kang T, Park SY, Kang CH, Lee SH, Park JH, Suh SW: Is biportal technique/endoscopic spinal surgery satisfactory for lumbar spinal stenosis patients?: A prospective randomized comparative study. Medicine (Baltimore) 98:e15451, 2019

11. Kim SK, Kang SS, Hong YH, Park SW, Lee SC: Clinical comparison of unilateral biportal endoscopic technique versus open microdiscectomy for single-level lumbar discectomy: A multicenter, retrospective analysis. J Orthop Surg Res 13:22, 2018

12. Pao JL, Lin SM, Chen WC, Chang CH: Unilateral biportal endoscopic decompression for degenerative lumbar canal stenosis. J Spine Surg 6:438-446, 2020

13. Park SM, Kim HJ, Kim GU, Choi MH, Chang BS, Lee CK, et al.: Learning curve for lumbar decompressive laminectomy in biportal endoscopic spinal surgery using the cumulative summation test for learning curve. World Neurosurg 122:e1007e1013, 2019

14. Pranata R, Lim MA, Vania R, July J: Biportal endoscopic spinal surgery versus microscopic decompression for lumbar spinal stenosis: A systematic review and meta-analysis. World Neurosurg 138:e450-e458, 2020

15. Sakane M: Anatomical relationship between Kambin's triangle and exiting nerve root. Mini-invasive Surgery, 2017

16. Takahashi H, Aoki Y, Saito J, Nakajima A, Sonobe M, Akatsu Y, et al.: Unilateral laminectomy for bilateral decompression improves low back pain while standing equally on both sides in patients with lumbar canal stenosis: Analysis using a detailed visual analogue scale. BMC Musculoskelet Disord 20:100, 2019 